\title{
ON PERIODIC SOLUTIONS OF AUTONOMOUS HAMILTONIAN SYSTEMS OF ORDINARY DIFFERENTIAL EQUATIONS
}

\author{
DAVID C. CLARK
}

\begin{abstract}
For the system $x^{\prime \prime}(t)+\operatorname{grad} U(x(t))=0$ lower bounds are obtained for the number of pairs $\pm x(t)$ of odd, periodic solutions, with the period prescribed. These bounds are in terms of the behavior of $U(x)$ near the origin and far away from the origin. It is assumed that $U(x)$ is even, and two different types of behavior of $U(x)$ far away from the origin are considered.
\end{abstract}

We seek to establish lower bounds for the number of odd solutions of a given period for the autonomous Hamiltonian system

$$
x^{\prime \prime}+\operatorname{grad} U(x)=0 \text {, }
$$

where $x: R \rightarrow R^{n}$ and $U$ is even and continuously differentiable. By a change of scale in the independent variable, we may normalize the problem so that we are seeking odd, $2 \pi$-periodic solutions of the system

$$
\alpha^{2} x^{\prime \prime}+\operatorname{grad} U(x)=0 .
$$

It is well known that if (1) is linear, there exist nontrivial solutions of (1) only for values of $\alpha$ in a certain discrete set. We shall be concerned with certain strictly nonlinear cases in which it turns out there are nontrivial solutions of (1) for values of $\alpha$ in a certain union of intervals. Thus it is reasonable to regard the value of $\alpha$ as given a priori.

Now we state some hypotheses and the main results.

(A1) $U(x)=\frac{1}{2} B x \cdot x+\rho(x)$, for $x \in R^{n}$, where $B$ is a constant symmetric matrix and $\rho(x)=o\left(|x|^{2}\right)$ as $|x| \rightarrow 0$.

(A2) $U(x) \leqq k_{1} \alpha^{2}|x|^{2}+k_{2}$, where $k_{1}<\frac{1}{2}$.

Let $\kappa_{1} \leqq \kappa_{2} \leqq \cdots \leqq \kappa_{n}$ be the eigenvalues of $B$.

THEOREM 1. Let (A1) and (A2) hold, and let $k$ be the number of pairs $(i, j)$ of positive integers such that $i^{2}<\kappa_{j} / \alpha^{2}$. Then there are at least $k$ distinct

Received by the editors January 18, 1972 and, in revised form, September 18, 1972 and November 20, 1972.

AMS (MOS) subject classifications (1970). Primary 34C25; Secondary 58E05.

Key words and phrases. Periodic solutions, Hamiltonian systems, LusternikSchnirelman theory, Palais-Smale condition, index of quadratic form.

(c) American Mathematical Society 1973 
pairs $\pm x(t)$ of nonidentically zero solutions of (1) which are odd and have period $2 \pi$.

(A3) $U(x)=\frac{1}{2} C x \cdot x+\sigma(x)$, where $C$ is a constant symmetric matrix and $|\operatorname{grad} \sigma(x)|=o(|x|)$ as $|x| \rightarrow \infty$.

Let $\mu_{1} \leqq \mu_{2} \leqq \cdots \leqq \mu_{n}$ be the eigenvalues of $C$.

(A4) $\mu_{j} / \alpha^{2} \neq k^{2}, j=1,2, \cdots, n ; k=1,2,3, \cdots$.

THEOREM 2. Let (A1), (A3), and (A4) hold. Let $k$ be as in Theorem 1 and let $l$ be the number of pairs $(i, j)$ of positive integers such that $i^{2}<\mu_{j} / \alpha^{2}$. If $k>l$, there are at least $k-l$ distinct pairs $\pm x(t)$ of nonidentically zero solutions of (1) which are odd and have period $2 \pi$.

The existence of periodic solutions of (1) has been studied before by a number of authors ([1], [2], [3], [6]), most recently by Berger ([1], [2]), who has, among other results, obtained a generalization of a theorem of Lyapunov. The feature by which the results of the present paper differ from previous results is that the period of the solutions is regarded as fixed a priori.

Proofs of Theorems 1 and 2. We shall reformulate the problem as one in the calculus of variations in a manner similar to that of Berger [1], and then we shall apply results of the author [5] on the Lusternik-Schnirelman theory of critical points of functionals.

Let $H$ denote the Hilbert space of odd, $2 \pi$-periodic, absolutely continuous functions $x: R \rightarrow R^{n}$, such that $x^{\prime}(t)$ is square integrable over $[0,2 \pi]$, where the inner product and norm are

Let

$$
(x, y)=\int_{0}^{2 \pi} x^{\prime}() \cdot y^{\prime}(t) d t, \quad\|x\|=(x, x)^{1 / 2} .
$$

$$
f(x)=\frac{1}{2} \alpha^{2}\|x\|^{2}-\int_{0}^{2 \pi} U(x(t)) d t .
$$

By standard regularity results, a critical point $x$ of $f$ relative to $H$ satisfies the Euler equation

$$
\alpha^{2} x^{\prime \prime}+\operatorname{grad} U(x)=r,
$$

where $r$ is even. But since $x(t)$ is odd, the left side of (3) is odd in view of (A1); hence $r(t)=0$. Thus the critical points of $f$ relative to $H$ are odd, $2 \pi$-periodic solutions of (1).

With this reduction to a problem in the calculus of variations, Theorems 1 and 2 are consequences of results in [5]. Before stating these results, we need some definitions.

Let $\mathscr{H}$ denote a real Hilbert space, $\phi(v)$ a real valued function on $\mathscr{H}$ 
with a continuous Fréchet derivative $\phi^{\prime}(v)$. The following condition is a variant of the Palais-Smale condition:

(C) Every bounded sequence $\left\{v_{k}\right\} \subset \mathscr{H}$ such that $\left\{\phi\left(v_{k}\right)\right\}$ is bounded and $\phi^{\prime}\left(v_{k}\right) \rightarrow 0$ contains a convergent subsequence $\left\{v_{k_{l}}\right\}$.

Let $q$ be a quadratic form on $\mathscr{H}$. If $k$ is the maximal dimension of subspaces of $\mathscr{H}$ on which $q$ is negative definite, we say that $q$ is of index $k$. ( $k=\infty$ is allowed.) $q$ is called regular if $q^{\prime}(v)$ exists and there exists $a>0$ such that $\left\|q^{\prime}(v)\right\| \geqq a\|v\|$ for all $v \in \mathscr{H}$.

THEOREM A. Let $\phi(v)$ be a real valued function on a real Hilbert space $\mathscr{H}$ such that $\phi$ is even, $C^{1}$, satisfies condition $(\mathrm{C})$, is bounded below, $\phi(v) \geqq 0$ for large $\|v\|$ and $\phi(v)=q(v)+o\left(\|v\|^{2}\right)$ as $\|v\| \rightarrow 0$, where $q$ is a quadratic form of index $k$. Then $\phi$ has at least $k$ pairs $\pm v$ of nonzero critical points.

THEOREM B. Let $\phi$ be a real valued function on a real Hilbert space $\mathscr{H}$ such that $\phi$ is even, $C^{1}$, satisfies condition $(\mathrm{C})$, is bounded below on bounded sets, $\phi(v)=q_{1}(v)+o\left(\|v\|^{2}\right)$ as $\|v\| \rightarrow 0$, where $q_{1}$ is a quadratic form of index $k$, and $\phi(v)=q_{2}(v)+r(x)$, where $q_{2}$ is a continuous, regular quadratic form of finite index $l$, and where $\left\|r^{\prime}(v)\right\|=o(\|v\|)$ as $\|v\| \rightarrow \infty$. If $k>l$, then $\phi$ has at least $k-l$ pairs $\pm v$ of nonzero critical points.

We now begin verifying the hypotheses of Theorems A and B for the particular cases involved in Theorems 1 and 2.

Since $U$ is even, $f$ is even. Since $U \in C^{1}\left(R^{n}\right)$, it is easily shown that $f$ has a locally uniform Fréchet derivative $f^{\prime}$ which is locally bounded. Hence $f^{\prime}$ is continuous [7, p. 43] and $f$ is $C^{1}$.

Also, since

$$
\sup _{0 \leqq t \leqq 2 \pi}|x(t)| \leqq \text { const }\|x\|, \quad \text { if } x \in H,
$$

and since $U \in C^{1}\left(R^{n}\right), f$ is bounded on bounded sets in $H$.

LemMa 1. $f$ satisfies condition (C) relative to $H$.

Proof. The proof is suggested by ideas of Browder, e.g. [4]. The only hypothesis used is that $U \in C^{1}\left(R^{n}\right)$. The Fréchet derivative $f^{\prime}(x)$ satisfies

$$
\left(f^{\prime}(x), y\right)=\alpha^{2}(x, y)-\int_{0}^{2 \pi} \operatorname{grad} U(x(t)) \cdot y(t) d t .
$$

Let $\left\{x^{(p)}\right\} \subset H$ be bounded and such that $f^{\prime}\left(x^{(p)}\right) \rightarrow 0$. It suffices to show that a subsequence of $\left\{x^{(p)}\right\}$ converges strongly. Since $\left\{x^{(p)}\right\}$ is bounded, a subsequence of $\left\{x^{(p)}\right\}$ converges weakly. We may assume that $\left\{x^{(p)}\right\}$ itself converges weakly. Denote the weak limit by $x$. We have that

$$
\lim _{p \rightarrow \infty}\left(f^{\prime}\left(x^{(p)}\right)-f^{\prime}(x), x^{(p)}-x\right)=0,
$$


since $f^{\prime}\left(x^{(p)}\right) \rightarrow 0$ and $x^{(p)} \rightarrow x$. On the other hand, by (5),

$$
\begin{aligned}
\left(f^{\prime}\left(x^{(p)}\right)-\right. & \left.f^{\prime}(x), x^{(p)}-x\right)=\alpha^{2}\left\|x^{(p)}-x\right\|^{2} \\
& \quad-\int_{0}^{2 \pi} \operatorname{grad} U\left(x^{(p)}(t)\right)-\operatorname{grad} U(x(t)) \cdot\left(x^{(p)}(t)-x(t)\right) d t .
\end{aligned}
$$

Since $x^{(p)} \rightarrow x, x^{(p)}(t) \rightarrow x(t)$ uniformly; hence by (7),

$$
\lim _{p \rightarrow \infty}\left(f^{\prime}\left(x^{(p)}\right)-f^{\prime}(x), x^{(p)}-x\right)=\alpha^{2} \lim _{p \rightarrow \infty}\left\|x^{(p)}-x\right\|^{2} .
$$

Hence, by (6), $\lim _{p \rightarrow \infty}\left\|x^{(p)}-x\right\|=0$, and so $\left\{x^{(p)}\right\}$ converges strongly. This completes the proof.

LEMMA 2. Under assumption (A1), $f(x)=q_{1}(x)+o\left(\|x\|^{2}\right)$ as $\|x\| \rightarrow 0$, where $q_{1}(x)$ is a quadratic form of index $k$ equal to the number of pairs $(i, j)$ of positive integers such that $i^{2}<\kappa_{j} / \alpha^{2}$.

Proof. By (A1) and (2), we may write $f(x)=q_{1}(x)+r_{1}(x)$, where

$$
q_{1}(x)=\frac{1}{2} \alpha^{2}\|x\|^{2}-\frac{1}{2} \int_{0}^{2 \pi} B x(t) \cdot x(t) d t, \quad r_{1}(x)=-\int_{0}^{2 \pi} \rho(x(t)) d t .
$$

By (A1) and (4), $r_{1}(x)=o\left(\|x\|^{2}\right)$ as $\|x\| \rightarrow 0$.

It remains to calculate the index of $q_{1}$. Let $u_{1}, u_{2}, \cdots, u_{n}$ be an orthonormal set of eigenvectors of $B$ corresponding to $\kappa_{1}, \kappa_{2}, \cdots, \kappa_{n}$, respectively. Let $H_{0}$ be the subspace of $H$ spanned by the functions $\sin (i t) u_{j}$, where $i^{2}<\kappa_{j} / \alpha^{2}$. Then it is easily shown that $q_{1}$ is negative definite on $H_{0}$ and positive semidefinite on $H_{0}^{\perp}$. Hence the index of $q_{1}$ is the dimension of $H_{0}$, which is the value $k$ defined in the lemma.

LEMMA 3. Under assumption (A2), $f(x) \geqq 0$ for large $\|x\|$.

Proof. By expansion of elements $x(t)$ of $H$ in Fourier sine series, it can be shown that

$$
\|x\|_{L_{2}} \leqq\|x\| \text {. }
$$

Hence, by (A2), $f(x) \geqq\left(\frac{1}{2}-k_{1} ; x^{2}\|x\|^{2}-k_{2} 2 \pi\right.$, so $f(x) \geqq 0$ for large $\|x\|$.

From Lemma 3 and the preceding remark that $f$ is bounded on bounded sets, it follows that if (A2) holds, $f$ is bounded below.

Theorem 1 follows from Theorem A, Lemmas 1 to 3 , and the preceding remarks in this section.

Under assumption (A3) we may write $f(x)=q_{2}(x)+r_{2}(x)$, where

$$
q_{2}(x)=\frac{1}{2} \alpha^{2}\|x\|^{2}-\frac{1}{2} \int_{0}^{2 \pi} C x(t) \cdot x(t) d t, \quad r_{2}(x)=-\int_{0}^{2 \pi} \sigma(x(t)) d t .
$$


LemMa 4. The quadratic form $q_{2}(x)$ is continuous in $H$, and has index $l$ equal to the number of pairs $(i, j)$ of positive integers such that $i^{2}<\mu_{j} / \alpha^{2}$. Under assumption (A4), $q_{2}(x)$ is regular.

Proof. Clearly $q_{2}(x)$ is bounded on the unit sphere in $H$, hence $q_{2}$ is continuous in $H$.

Let $u_{1}, u_{2}, \cdots, u_{n}$ be an orthonormal set of eigenvectors corresponding to $\mu_{1}, \mu_{2}, \cdots, \mu_{n}$ respectively. Let $H_{0}$ be the subspace of $H$ spanned by $\sin (i t) u_{j}$ such that $i^{2}<\mu_{j} / \alpha^{2}$. Then $H_{0}^{\perp}$ is spanned by $\sin (i t) u_{j}$ such that $i^{2}>\mu_{j} / \alpha^{2}$ in view of (A4). Also $q_{2}$ is negative definite on $H_{0}$ and positive definite on $H_{0}^{\perp}$. The dimension of $H_{0}$ is $l$, and is clearly the index of $q_{2}$.

We have that $q_{2}(x)=\frac{1}{2} \beta(x, x)$, where

$$
(x, y)=\alpha^{2}(x, y)-\int_{0}^{2 \pi} C x(t) \cdot y(t) d t .
$$

$\beta$ is a symmetric bilinear form. Also, $\left(q_{2}^{\prime}(x), y\right)=\beta(x, y)$. Hence

$$
\left\|q_{2}^{\prime}(x)\right\| \geqq|\beta(x, y)| /\|y\| \text {. }
$$

It is easily shown that

$$
\beta(u, v)=0 \quad \text { if } u \in H_{0}, v \in H_{0}^{\perp} .
$$

For any $x \in H$, we write $x=u+v, u \in H_{0}, v \in H_{0}^{\perp}$. Using (9) and (10),

$$
\begin{aligned}
& \left\|q_{2}^{\prime}(x)\right\| \geqq|\beta(u, u)| /\|u\|=2\left|q_{2}(u)\right| /\|u\|, \\
& \left\|q_{2}^{\prime}(x)\right\| \geqq|\beta(v, v)| /\|v\|=2\left|q_{2}(v)\right| /\|v\|,
\end{aligned}
$$

if $u \neq 0$ and $v \neq 0$. It is clear that $\left|q_{2}(u)\right| /\|u\|^{2}$ and $\left|q_{2}(v)\right| /\|v\|^{2}$ are bounded below for $0 \neq u \in H_{0}$ and $0 \neq v \in H_{0}^{\perp}$. Hence, from (11),

$$
\left\|q_{2}^{\prime}(x)\right\| \geqq \max (c\|u\|, c\|v\|)
$$

for some $c>0$. Since either $\|u\| \geqq \frac{1}{2}\|x\|$ or $\|v\| \geqq \frac{1}{2}\|x\|$, it follows from (12) that $q_{2}$ is regular.

LEMMA 5. Under assumption (A3), $r_{2}^{\prime}(x)=o(\|x\|)$ as $\|x\| \rightarrow \infty$.

Proof. We have the equality

$$
\left(r_{2}^{\prime}(x), y\right)=-\int_{0}^{2 \pi} \operatorname{grad} \sigma(x(t)) \cdot y(t) d t .
$$

Setting $y=r_{2}^{\prime}(x)$ and using inequality (8),

$$
\left\|r_{2}^{\prime}(x)\right\| \leqq\|\operatorname{grad} \sigma(x(t))\|_{L_{2}} .
$$

It follows from (A3) that $\|\operatorname{grad} \sigma(x(t))\|_{L_{2}}=o\left(\|x\|_{L_{2}}\right)$ as $\|x\|_{L_{2}} \rightarrow \infty$. Hence, from (8) and (13), $\left\|r_{2}^{\prime}(x)\right\|=o(\|x\|)$ as $\|x\| \rightarrow \infty$. 
Theorem 2 follows from Theorem B, Lemmas 1, 2, 4, and 5, and the remarks in this section.

\section{BIBLIOGRAPHY}

1. M. S. Berger, On periodic solutions of second order Hamiltonian systems, J. Math. Anal. Appl. 29 (1970), 512-522. MR 41 \#2121.

2. - Periodic solutions of second order dynamical systems and isoperimetric variational problems, Amer. J. Math. 93 (1971), 1-10. MR 43 \#2588.

3. G. D. Birkhoff, Dynamical systems, Amer. Math. Soc. Colloq. Publ., vol. 9, Amer. Math. Soc., Providence, R.I., 1927.

4. F. E. Browder, Existence theorems for nonlinear partial differential equations, Proc. Sympos. Pure Math., vol. 16, Amer. Math. Soc., Providence, R.I., 1970, pp. 1-60. MR 42 \#4855.

5. D. C. Clark, A variant of the Lusternik-Schnirelman theory, Indiana Univ. Math. J. 22 (1972), 65-74.

6. V. Nemyckiř and V. Stepanov, Qualitative theory of differential equations, OGIZ, Moscow, 1947; English transl., Princeton Math. Series, no. 22, Princeton Univ. Press, Princeton, N.J., 1960. MR 10, 612; MR 22 \#12258.

7. M. M. Vaĭnberg, Variational methods for the study of nonlinear operators, GITTL, Moscow, 1956; English transl., Holden-Day, San Francisco, Calif., 1964. MR 19, 567; MR 31 \#638.

Department of Mathematics, University of Puerto Rico, Mayaguez, Puerto Rico 00708 